PROCEEDINGS OF THE AMERICAN MATHEMATICAL SOCIETY

Volume 124, Number 5, May 1996

\title{
A NOTE ON FUCHS' PROBLEM 34
}

\author{
U. F. ALBRECHT AND H. P. GOETERS
}

(Communicated by Ronald M. Solomon)

\begin{abstract}
We investigate to what extent an abelian group $G$ is determined by the homomorphism groups $\operatorname{Hom}(G, B)$ where $B$ is chosen from a set $\mathcal{X}$ of abelian groups. In particular, we address Problem 34 in Professor Fuchs' book which asks if $\mathcal{X}$ can be chosen in such a way that the homomorphism groups determine $G$ up to isomorphism. We show that there is a negative answer to this question. On the other hand, there is a set $\mathcal{X}$ which determines the torsion-free groups of finite rank up to quasi-isomorphism.
\end{abstract}

\section{INTRODUCTION}

Of fundamental importance in the study of abelian groups is the structure of the homomorphism group $\operatorname{Hom}(A, G)$. Naturally one would like to know the extent to which $A$ is determined by the structure of the groups $\operatorname{Hom}(A, G)$ where $G$ is chosen from a given set $\mathcal{X}$ of abelian groups. In particular, is it possible to choose a set $\mathcal{X}$ in such a way that $A$ is determined up to isomorphism by the groups $\operatorname{Hom}(A, G)$ for $G \in \mathcal{X}$, as has been asked in Problem 34 of [4]? The subject of this paper is to address these questions for $p$-groups and torsion-free groups of finite rank.

The first result of Section 2 shows that it is not possible to give a positive answer to [4, Problem 34]: For any set $\mathcal{X}$ of abelian groups, there are non-isomorphic reduced abelian groups $A$ and $C$ such that $\operatorname{Hom}(A, G) \cong \operatorname{Hom}(C, G)$ for all $G \in \mathcal{X}$. We, therefore, turn to the problem of describing properties shared by abelian groups $A$ and $C$ for which $\operatorname{Hom}(A, G) \cong \operatorname{Hom}(C, G)$ for all $G$ in a given set of abelian groups. In Theorem 2.2, we characterize those collections $\mathcal{X}$ of abelian $p$-groups such that the groups $\operatorname{Hom}(A, G)$ for $G \in \mathcal{X}$ determine the finite Ulm-Kaplansky invariants $\left\{f_{n}(A) \mid n<\omega\right\}$. Unfortunately, the characterization is valid only under the additional assumption that the weak Continuum Hypothesis $\left(2^{\kappa}=2^{\lambda} \Rightarrow \kappa=\lambda\right)$ holds. We show that there exists a model of ZFC in which this characterization fails (Corollary 2.6). As a consequence of the proof of Corollary 2.6, we obtain that there are non-isomorphic abelian groups $A$ and $C$ such that, for any set $\mathcal{X}$ of abelian groups, there is a model of ZFC in which $\operatorname{Hom}(A, G) \cong \operatorname{Hom}(C, G)$ for all $G \in \mathcal{X}$

Section 3 addresses Problem 34 for torsion-free groups of finite rank. The restriction to finite rank is necessary to avoid set-theoretic problems similar to those described in the context of $p$-groups. Our arguments are general enough to hold for

Received by the editors October 1, 1993 and, in revised form, December 12, 1993.

1991 Mathematics Subject Classification. Primary 20K15, 20K30; Secondary 20J05.

Key words and phrases. Homomorphism group, Martin's Axiom, p-group, torsion-free group.

(C)1996 American Mathematical Society 
modules over integral domains. We want to remind the reader that the concepts of quasi-isomorphism and quasi-equality carry over from torsion-free abelian groups to torsion-free modules over an integral domain $R$. Theorem 3.2 shows that two torsion-free $R$-modules $A$ and $C$ whose ranks do not exceed $m<\omega$ are determined up to quasi-isomorphism by the modules $\operatorname{Hom}_{R}(A, G)$ where $G \in \mathcal{X}=\{G \mid G$ is torsion-free and $\left.r_{0}(G) \leq m\right\}$. Example 3.5 shows that the set $\mathcal{X}$ does not determine $A$ and $C$ up to isomorphism in general.

\section{A negative Answer to Fuchs' Problem 34}

The first result of this section shows that there is a negative answer to [4, Problem 34]. Since the groups $A$ and $C$ which are to be constructed will be $p$-groups, it is enough to restrict our attention to the case that $\mathcal{X}$ is a set of abelian $p$ groups. In the following, we write $f_{\sigma}(A)$ for the $\sigma$ th-Ulm-Kaplansky-Invariant $\operatorname{dim}_{\mathbb{Z} / p \mathbb{Z}} p^{\sigma} A[p] / p^{\sigma+1} A[p]$ of an abelian $p$-group $A$.

Theorem 2.1. For any set $\mathcal{X}$ of reduced abelian p-groups, there exist two nonisomorphic, totally projective p-groups $A_{1}$ and $A_{2}$ such that the groups $\operatorname{Hom}\left(A_{1}, G\right)$ and $\operatorname{Hom}\left(A_{2}, G\right)$ are isomorphic for all $G \in \mathcal{X}$.

Proof. Let $\tau=\sup \{\operatorname{length}(G) \mid G \in \mathcal{X}\}$, and choose a cardinal $\kappa>\tau+\omega$. For an ordinal $\sigma<\tau$, define $g_{1}(\sigma)=g_{2}(\sigma)=\kappa$. For $n<\omega$, set $g_{1}(\tau+n)=\aleph_{0}$ if $n$ is even, and $g_{1}(\tau+n)=0$ if $n$ is odd. Similarly, $g_{2}(\tau+n)=0$ for even $n$ and $\aleph_{0}$ for odd $n$. Clearly, $\sup \left\{\sigma+1 \mid g_{i}(\sigma) \neq 0\right\}=\tau+\omega$. Moreover, if $\sigma$ is an ordinal with $\sigma+\omega<\tau+\omega$, then $\sigma<\tau$ and $\Sigma_{n<\omega} g_{i}(\sigma+n) \geq g_{i}(\sigma)=\kappa$ while $\Sigma_{\rho \geq \sigma+\omega} g_{i}(\rho) \leq \kappa$. This shows that $g_{1}$ and $g_{2}$ are $\tau+\omega$-admissible functions. By [4, Theorem 83.6], there are non-isomorphic totally projective $p$-groups $A_{1}$ and $A_{2}$ of length $\tau+\omega$ such that $f_{\sigma}\left(A_{i}\right)=g_{i}(\sigma)$ for all $\sigma<\tau+\omega$.

Observe that $A_{1}$ and $A_{2}$ have $p$-basic subgroups $B_{1}^{\prime}$ and $B_{2}^{\prime}$ isomorphic to $\bigoplus_{n<\omega} \bigoplus_{\kappa} \mathbb{Z} / p^{n} \mathbb{Z}$. Since $\bigoplus_{n<\omega} \mathbb{Z} / p^{n} \mathbb{Z}$ contains a proper $p$-basic subgroup, we can find a $p$-basic subgroup $B_{i}$ of $B_{i}^{\prime}$ with $B_{i}^{\prime} / B_{i} \cong \bigoplus_{\kappa} \mathbb{Z}\left(p^{\infty}\right)$. Since $\left|A_{i}\right|=$ $\Sigma_{\sigma<\tau+\omega} g_{i}(\sigma)=\kappa$, we have $A_{i} / B_{i} \cong \bigoplus_{\kappa} \mathbb{Z}\left(p^{\infty}\right)$. If $D$ is a divisible $p$-group, then

$$
\begin{aligned}
\operatorname{Hom}\left(A_{i}, D\right) & \cong \operatorname{Hom}\left(B_{i}, D\right) \oplus \operatorname{Hom}\left(A_{i} / B_{i}, D\right) \\
& \cong \operatorname{Hom}\left(B_{i}, D\right) \oplus\left[\prod_{\kappa} \operatorname{Hom}\left(\mathbb{Z}\left(p^{\infty}\right), D\right)\right] .
\end{aligned}
$$

Therefore, $\operatorname{Hom}\left(A_{1}, D\right) \cong \operatorname{Hom}\left(A_{2}, D\right)$.

Let $G \in \mathcal{X}$. Write $G=D \oplus E$ such that $D$ is divisible and $E$ is reduced. By what has been shown so far, it is enough to establish $\operatorname{Hom}\left(A_{1}, E\right) \cong \operatorname{Hom}\left(A_{2}, E\right)$. The choice of $\tau$ guarantees that $E$ is a reduced $p$-group of length at most $\tau$. Therefore, $\operatorname{Hom}\left(A_{i}, E\right) \cong \operatorname{Hom}\left(A_{i} / p^{\tau} A_{i}, E\right)$. Since $A_{1} / p^{\tau} A_{1} \cong A_{2} / p^{\tau} A_{2}$, we indeed have $\operatorname{Hom}\left(A_{1}, E\right) \cong \operatorname{Hom}\left(A_{2}, E\right)$.

We now investigate properties shared by $p$-groups $A$ and $C$ for which $\operatorname{Hom}(A, G)$ $\cong \operatorname{Hom}(C, G)$ for all $G$ in a family $\mathcal{X}$ of $p$-groups. Our first result shows that the finite Ulm-Kaplansky invariants can be recovered in this way:

Theorem 2.2. Consider the following conditions for a class $\mathcal{X}$ of p-groups:

(a) If abelian p-groups $A$ and $C$ satisfy $\operatorname{Hom}(A, G) \cong \operatorname{Hom}(C, G)$ for all $G \in \mathcal{X}$, then $f_{n}(A)=f_{n}(C)$ for all $n<\omega$. 
(b) For every $0<n<\omega$, there is a group $G \in \mathcal{X}$ with $0<\left|p^{n} G[p]\right|<\infty$ and $f_{n-1}(G)=0$.

Then, (a) always implies (b), and the converse holds if one assumes the weak GCH, i.e. $\kappa<\lambda$ implies $2^{\kappa}<2^{\lambda}$ for all cardinals $\kappa$ and $\lambda$.

Proof. Let $G$ be any abelian $p$-group with $p^{n} G[p]$ finite; and choose a $p$-basic subgroup $B=\bigoplus_{n=1}^{\infty} B_{n}$ of $G$ where $B_{n} \cong \bigoplus_{I_{n}} \mathbb{Z}\left(p^{n}\right)$ for some index-set $I_{n}$. Then, $G=B_{1} \oplus \cdots \oplus B_{n} \oplus D$ for some subgroup $D$ of $G$. Since $D$ has no cyclic summands of order less than $p^{n+1}$ and $p^{n} D[p]$ is finite, $D=T \oplus\left[\bigoplus_{m} \mathbb{Z}\left(p^{\infty}\right)\right]$ where $T$ is a finite group and $m<\omega$.

(a) $\Rightarrow$ (b): Suppose that (b) fails for the positive integer $n$. We consider the groups $A_{i}=\bigoplus_{\omega} \mathbb{Z}\left(p^{i}\right)$ for $i=1, \ldots, n-1$ and $i=n+1$. Set $A=A_{1} \oplus \cdots \oplus$ $A_{n-1} \oplus \mathbb{Z}\left(p^{n}\right) \oplus A_{n+1}$ and $C=A \oplus \mathbb{Z}\left(p^{n}\right)$. Since $f_{n-1}(A)=1$ and $f_{n-1}(C)=$ 2 , there is a group $G \in \mathcal{X}$ with $\operatorname{Hom}(A, G) \neq \operatorname{Hom}(C, G)$. If $p^{n} G[p]=0$, then $p^{n} G=0$, so that any map from $A_{n+1}$ into $G$ contains $p^{n} A=p^{n} A_{n+1}$ in its kernel. Consequently, $\operatorname{Hom}(A, G) \cong \operatorname{Hom}\left(A^{\prime} \oplus\left(A_{n+1} / p^{n} A_{n+1}\right), G\right)$ and $\operatorname{Hom}(C, G) \cong \operatorname{Hom}\left(C^{\prime} \oplus\left(A_{n+1} / p^{n} A_{n+1}\right), G\right)$ where $A^{\prime}=A_{1} \oplus \cdots \oplus A_{n-1} \oplus \mathbb{Z}\left(p^{n}\right)$ and $C^{\prime}=A^{\prime} \oplus \mathbb{Z}\left(p^{n}\right)$. However, $A^{\prime} \oplus\left(A_{n+1} / p^{n} A_{n+1}\right) \cong C^{\prime} \oplus\left(A_{n+1} / p^{n} A_{n+1}\right)$, so that $G\left[p^{n}\right]=0$ is impossible. Suppose that $p^{n} G[p]$ is infinite, and choose a $p$-basic subgroup $B=\bigoplus_{n=1}^{\infty} B_{n}$ of $G$ where each $B_{n}$ is a direct sum of cyclics of order $p^{n}$. We write $G=B_{1} \oplus \cdots \oplus B_{n} \oplus E$ for some subgroup $E$ of $G$. Observe $\operatorname{Hom}\left(A_{i}, G\right) \cong \Pi_{\omega} \operatorname{Hom}\left(\mathbb{Z}\left(p^{i}\right), G\right) \cong \Pi_{\omega} G\left[p^{i}\right]$ for $i=1, \ldots, n-1, n+1$ and $\operatorname{Hom}\left(\mathbb{Z}\left(p^{n}\right), G\right) \cong G\left[p^{n}\right]=B_{1} \oplus \cdots \oplus B_{n} \oplus E\left[p^{n}\right]$. We can write $E\left[p^{n}\right]=L_{1} \oplus \cdots \oplus L_{n}$ where each $L_{i}$ is a direct sum of cyclic groups of order $p^{i}$. By Szele's Theorem, $B_{i} \oplus L_{i}$ is a direct summand of $G\left[p^{i}\right]$, and hence $\Pi_{\omega} G\left[p^{i}\right] \oplus B_{i} \oplus L_{i} \cong \Pi_{\omega} G\left[p^{i}\right]$. This shows $\operatorname{Hom}(A, G) \cong \Pi_{\omega}\left[\bigoplus_{i=1}^{n-1} G\left[p^{i}\right] \oplus G\left[p^{n+1}\right]\right] \oplus B_{n} \oplus L_{n}$ while $\operatorname{Hom}(C, G) \cong$ $\operatorname{Hom}(A, G) \oplus B_{n} \oplus L_{n}$. Once we have shown that $B_{n} \oplus L_{n}$ is infinite, we obtain $\left(B_{n} \oplus L_{n}\right)^{2} \cong B_{n} \oplus L_{n}$ and $\operatorname{Hom}(A, G) \cong \operatorname{Hom}(C, G)$.

If $B_{n} \oplus E$ has an infinite $p$-basic subgroup $B^{\prime}$, then $B^{\prime}$ has no cyclic direct summands of order less than $p^{n}$. In particular, $B^{\prime}\left[p^{n}\right]$ is an infinite direct sum of groups isomorphic to $\mathbb{Z}\left(p^{n}\right)$. By Szele's Theorem, $B^{\prime}\left[p^{n}\right]$ is a direct summand of $G\left[p^{n}\right]$, which is only possible if $B_{n} \oplus L_{n}$ is infinite. If $B_{n} \oplus E$ has a finite $p$-basic subgroup, then $B_{n} \oplus E \cong T \oplus \bigoplus_{I} \mathbb{Z}\left(p^{\infty}\right)$ for some index-set $I$ and some finite group $T$. If $I$ were finite, then $p^{n} G=p^{n} E$ would have a finite $p$-socle, contrary to our assumption. Thus, $I$ is infinite, and $B_{n} \oplus E$ contains a subgroup $D$ which is an infinite direct sum of cyclic groups of order $p^{n}$. As before, $D$ is a direct summand of $G\left[p^{n}\right]$, and $B_{n} \oplus L_{n}$ is infinite. Consequently, $p^{n} G[p]$ is finite.

Since (b) fails for $n$ and $0<\left|p^{n} G[p]\right|<\infty$, we have $f_{n-1}(G) \neq 0$. Since $E$ has no cyclic summands of order less than $p^{n+1}$, we obtain $p^{n-1} E[p]=p^{n} E[p]$. Therefore, $p^{n-1} G[p] / p^{n} G[p] \cong p^{n-1} B_{n}[p]$ is non-zero. By the initial remarks to this proof, $E$ is finitely cogenerated; and $L_{n}$ is finite. We write $G\left[p^{n+1}\right]=B_{1} \oplus \cdots \oplus B_{n} \oplus$ $K_{1} \oplus \cdots \oplus K_{n+1}$ where $K_{i} \cong \bigoplus_{J_{i}} \mathbb{Z}\left(p^{i}\right)$ is a subgroup of $E$. Since $B_{n} \neq 0$, we have $\left[\Pi_{\omega} B_{n}\right] \oplus B_{n} \oplus L_{n} \cong \Pi_{\omega} B_{n}$, and $\left[\Pi_{\omega} G\left[p^{n+1}\right]\right] \oplus B_{n} \oplus L_{n} \cong \Pi_{\omega} G\left[p^{n+1}\right]$. This shows $\operatorname{Hom}(A, G) \cong \Pi_{\omega}\left[\bigoplus_{i=1}^{n-1} G\left[p^{i}\right] \oplus G\left[p^{n+1}\right]\right] \cong \operatorname{Hom}(C, G)$. The resulting contradiction establishes (b).

(b) $\Rightarrow$ (a): Assume the weak continuum hypotheses. Let $A$ and $C$ be abelian $p$-groups with $\operatorname{Hom}(A, G) \cong \operatorname{Hom}(C, G)$ for all $G \in \mathcal{X}$. Choose a $p$-basic subgroup $B=\bigoplus_{n=1}^{\infty} B_{n}$ of $A$ with $B_{n}$ a direct sum of cyclics of order $p^{n}$. If $B_{n} \bigoplus_{\sigma_{n}} \mathbb{Z}\left(p^{n}\right)$, then $f_{n-1}(A)=\sigma_{n}$. Choose a group $G \in \mathcal{X}$ with $0<\left|p^{n} G[p]\right|<\infty$ and $f_{n-1}(G)=$ 
0 . By the initial remarks of this proof, $G=D_{0} \oplus\left[\bigoplus_{i=1}^{m} D_{i}\right] \oplus S$ where $p^{n-1} D_{0}=0$, $D_{i} \cong \bigoplus_{t_{i}} \mathbb{Z}\left(p^{s_{i}}\right)$ with integers $t_{i}>0$ and $s_{i}>n$ for $i=1, \ldots, m$, and $S \cong$ $\bigoplus_{r} \mathbb{Z}\left(p^{\infty}\right)$ for some $r<\omega$. Observe that $G$ has no direct summand isomorphic to $\mathbb{Z}\left(p^{n}\right)$ since $f_{n-1}(G)=0$. We now determine the $(n-1)^{s t}$-Ulm-Kaplansky-invariant of the torsion subgroup $t \operatorname{Hom}(A, G)$ of $\operatorname{Hom}(A, G)$ :

Write $A=B_{1} \oplus \cdots \oplus B_{n} \oplus E$, and obtain a decomposition

$$
\operatorname{Hom}(A, G) \cong \operatorname{Hom}\left(B_{1} \oplus \cdots \oplus B_{n-1}, G\right) \oplus \operatorname{Hom}\left(B_{n} \oplus E, G\right) .
$$

Since the first summand in this direct sum is annihilated by $p^{n-1}$, we have

$$
f_{n-1}(t \operatorname{Hom}(A, G))=f_{n-1}\left(t \operatorname{Hom}\left(B_{n} \oplus E, G\right)\right)
$$

is the sum of $f_{n-1}\left(\operatorname{Hom}\left(B_{n}, \bigoplus_{i=1}^{m} D_{i} \oplus S\right)\right)$ and $f_{n-1}\left(t \operatorname{Hom}\left(E, \bigoplus_{i=1}^{m} D_{i} \oplus S\right)\right)$. To show that the second term vanishes, we observe that

$$
\operatorname{Hom}\left(E, D_{i}\right) \cong \operatorname{Hom}\left(E / p^{s_{i}} E, D_{i}\right) \cong \operatorname{Hom}\left(\bigoplus_{j=n+1}^{\infty} B_{j} / p^{s_{i}} B_{j}, D_{j}\right)
$$

is bounded, and hence a direct sum of cyclic groups of order at least $p^{n+1}$. Therefore, $f_{n-1}\left(\operatorname{Hom}\left(E, D_{i}\right)\right)=0$. If $S \neq 0$, then

$$
\operatorname{Hom}(E, S) \cong \bigoplus_{r}\left[\Pi_{j \geq n+1}\left(\Pi_{L_{j}} \mathbb{Z}\left(p^{j}\right)\right) \oplus \Pi_{L} J_{p}\right]
$$

for index-sets $L$ and $L_{n+1}, \ldots$ Therefore, $\bigoplus_{r}\left[\bigoplus_{j \geq n+1}\left(\Pi_{L_{j}} \mathbb{Z}\left(p^{j}\right)\right)\right]$ is a $p$-basic subgroup of $t \operatorname{Hom}(E, S)$. Consequently, $f_{n-1}(t \operatorname{Hom}(E, S))=0$. This shows

$$
\begin{aligned}
f_{n-1}(t \operatorname{Hom}(A, G)) & =f_{n-1}\left(\operatorname{Hom}\left(B_{n}, \bigoplus_{i=1}^{m} D_{i} \oplus S\right)\right) \\
& = \begin{cases}\sigma_{n}\left(t_{1}+\cdots+t_{m}+r\right) & \text { if } \sigma_{n} \text { is finite } \\
2^{\sigma_{n}} & \text { otherwise. }\end{cases}
\end{aligned}
$$

If $f_{n-1}(C)=\tau_{n}$, then a similar computation shows that $\sigma_{n}$ is infinite if and only if $\tau_{n}$ is. For finite values of $\sigma_{n}$, we have $\sigma_{n}=\tau_{n}$ since $t_{1}+\cdots+t_{n}+r \neq 0$. If $\sigma_{n}$ is infinite, then $2^{\sigma_{n}}=2^{\tau_{n}}$ yields $\sigma_{n}=\tau_{n}$ because we assume the weak GCH.

The weak GCH in the proof of implication $(b) \Rightarrow(a)$ in the last result can be removed if $\mathcal{X}$ contains all bounded $p$-groups. We want to remind the reader that a homomorphism $\alpha: G \rightarrow H$ between $p$-groups is small if, for every $m<\omega$, there is $n<\omega$ such that $\alpha\left(p^{n} A\left[p^{m}\right]\right)=0$. The collection of all small homomorphisms between $G$ and $H$ forms a subgroup of $\operatorname{Hom}(G, H)$ which is denoted by $\operatorname{Hom}_{S}(G, H)$.

Theorem 2.3. Let $\mathcal{X}$ be a class of p-groups which contains all bounded p-groups. The following conditions are equivalent for abelian p-groups $A$ and $C$ :

(a) $f_{n}(A)=f_{n}(C)$ for all $n<\omega$.

(b) $\operatorname{Hom}(A, G) \cong \operatorname{Hom}(C, G)$ for all torsion-complete p-groups $G$.

(c) $\operatorname{Hom}_{S}(A, G) \cong \operatorname{Hom}_{S}(C, G)$ for all $G \in \mathcal{X}$.

(d) $t \operatorname{Hom}(A, G) \cong t \operatorname{Hom}(C, G)$ for all $G \in \mathcal{X}$.

Proof. (a) $\Rightarrow$ (b): If $B$ is a $p$-basic subgroup of $A$, then $B=\bigoplus_{n=1}^{\infty} B_{n}$ with $B_{n} \cong$ $\bigoplus_{f_{n-1}(A)} \mathbb{Z}\left(p^{n}\right)$. Since torsion-complete groups are injective with respect to pureexact sequences of abelian $p$-groups, we obtain an exact sequence $\operatorname{Hom}(A / B, G) \rightarrow$ $\operatorname{Hom}(A, G) \rightarrow \operatorname{Hom}(B, G) \rightarrow 0$. If $G$ is torsion-complete, then $G$ is reduced so that 
$\operatorname{Hom}(A / B, G)=0$. Therefore, $\operatorname{Hom}(A, G)$ is completely determined by $B$. Since $A$ and $C$ have isomorphic $p$-basic subgroups, we have $\operatorname{Hom}(A, G) \cong \operatorname{Hom}(C, G)$.

(a) $\Rightarrow(\mathrm{c})$ : Let $G$ be in $\mathcal{X}$. As before, let $B$ be a $p$-basic subgroup of $A$. We consider the induced exact sequence $0 \rightarrow \operatorname{Hom}(A / B, G) \rightarrow \operatorname{Hom}(A, G) \stackrel{\alpha}{\rightarrow} \operatorname{Hom}(B, G)$ in which $\alpha$ is the restriction map. Clearly, $\alpha\left(\operatorname{Hom}_{S}(A, G)\right) \subseteq \operatorname{Hom}_{S}(B, G)$. By [4], for every small homomorphism $\sigma: B \rightarrow G$, there is a small homomorphism $\tau: A \rightarrow G$ with $\tau \mid B=\sigma$. Hence, $\alpha\left(\operatorname{Hom}_{S}(A, G)\right)=\operatorname{Hom}_{S}(B, G)$. Let $\sigma: A \rightarrow G$ be a small homomorphism with $\sigma(B)=0$. For any $m<\omega$, there is an $n<\omega$ with $\sigma\left(p^{n} A\left[p^{m}\right]\right)=0$. Let $a \in A\left[p^{m}\right]$. Since $p^{n} A+B=A$, we can write $a=p^{n} a^{\prime}+b$ for some $a^{\prime} \in A$ and $b \in B$. We have $p^{n+m} a^{\prime}+p^{m} b=0$. Therefore, there is $b^{\prime} \in B$ with $p^{n+m} a^{\prime}=p^{n+m} b^{\prime}$. Then, $p^{n} a^{\prime}-p^{n} b^{\prime} \in p^{n} A\left[p^{m}\right]$. Thus, $\sigma(a)=$ $\sigma\left(p^{n} a^{\prime}-p^{n} b^{\prime}\right)+\sigma\left(b+p^{n} b^{\prime}\right)=0$. This shows that $\alpha: \operatorname{Hom}_{S}(A, G) \rightarrow \operatorname{Hom}_{S}(B, G)$ is an isomorphism, which establishes $\operatorname{Hom}_{S}(A, G) \cong \operatorname{Hom}_{S}(C, G)$.

(c) $\Rightarrow$ (d): Since every torsion element of $\operatorname{Hom}(A, G)$ is a small homomorphism, the given isomorphism $\operatorname{Hom}_{S}(A, G) \cong \operatorname{Hom}_{S}(C, G)$ induces the desired isomorphism between $t \operatorname{Hom}(A, G)$ and $t \operatorname{Hom}(C, G)$.

(b) or (d) $\Rightarrow$ (a): Suppose that one of the two conditions holds, and let $n$ be given. For a chosen cardinal $\kappa$, let $G=\bigoplus_{\kappa} \mathbb{Z}\left(p^{n+1}\right)$, an element of $\mathcal{X}$. Then, $\operatorname{Hom}(A, G)$ is bounded, and hence $\operatorname{Hom}(A, G) \cong \operatorname{Hom}(C, G)$. In a way similar to that used in the proof of implication (b) $\Rightarrow$ (a) of Theorem 2.2, we obtain

$$
f_{n}(\operatorname{Hom}(A, G))= \begin{cases}f_{n}(A) \kappa & \text { if } f_{n}(A) \text { is finite } \\ \kappa^{f_{n}(A)} & \text { otherwise. }\end{cases}
$$

If $f_{n}(A)$ is finite, we choose $\kappa$ to be finite, from which $f_{n}(A)=f_{n}(C)$ follows. We now consider the case that $f_{n}(A)$ and $f_{n}(C)$ are infinite. Without loss of generality, we may assume $f_{n}(A) \leq f_{n}(C)$. Let $\kappa$ be a strong limit cardinal with $\operatorname{cf}(\kappa)=\left(f_{n}(A)\right)^{+}$and $f_{n}(C)<\kappa$. For every $\lambda<\kappa$ we have $\lambda^{f_{n}(C)}<\kappa$ since $\kappa$ is a strong limit cardinal. Therefore, $\kappa^{f_{n}(C)}=\kappa^{\mathrm{cf}(\kappa)}>\kappa$ by König's Theorem. On the other hand, if $f_{n}(A)<f_{n}(C)$, then $\kappa^{f_{n}(A)}=\kappa$. This shows $\operatorname{Hom}(A, G) \not$ $\operatorname{Hom}(C, G)$, a contradiction. Thus, $f_{n}(A)=f_{n}(C)$.

As a consequence of the arguments used in the proof of the last implication of Theorem 2.3, we obtain

Corollary 2.4. Let $A$ and $C$ be either both direct sums of cyclics or both torsioncomplete. The following statements are equivalent:

(a) $A \cong C$.

(b) $\operatorname{Hom}(A, G) \cong \operatorname{Hom}(C, G)$ for all p-groups $G$ which are direct sums of cyclics.

(c) $\operatorname{Hom}(A, G) \cong \operatorname{Hom}(C, G)$ for all p-groups $G$.

However, without some immediate restrictions on the structure of $A$ as in Corollary 2.4, it is impossible to show that $A$ is determined up to isomorphism by the structure of the groups $\{\operatorname{Hom}(A, G) \mid G$ is a $p$-group $\}$.

Example 2.5. There exist abelian p-groups $A$ and $C$ such that $f_{n}(A)=f_{n}(C)$ for all $n<\omega$, but $\operatorname{Hom}(A, G) \varsubsetneqq \operatorname{Hom}(C, G)$ for some p-group $G$.

Proof. Let $A=\bigoplus_{n=1}^{\infty} \mathbb{Z}\left(p^{n}\right)$ and $C$ be the torsion-complete group with $p$-basic subgroup $A$. Then, $\operatorname{Hom}\left(A, \mathbb{Z}\left(p^{\infty}\right)\right) \cong \prod_{n=1}^{\infty} \mathbb{Z}\left(p^{n}\right)$ has cardinality $2^{\aleph_{0}}$. On the other hand, $\operatorname{Hom}\left(C, \mathbb{Z}\left(p^{\infty}\right)\right)$ contains $\operatorname{Hom}\left(C / A, \mathbb{Z}\left(p^{\infty}\right)\right)$ as a subgroup. Since 
$C / A \cong \bigoplus_{2^{\aleph_{0}}} \mathbb{Z}\left(p^{\infty}\right)$, we have $\left|\operatorname{Hom}\left(C, \mathbb{Z}\left(p^{\infty}\right)\right)\right| \geq\left|\Pi_{2^{\aleph_{0}}} J_{p}\right|=2^{2^{\aleph_{0}}}$. Therefore, $\operatorname{Hom}\left(A, \mathbb{Z}\left(p^{\infty}\right)\right) \not \operatorname{Hom}\left(C, \mathbb{Z}\left(p^{\infty}\right)\right)$, while $f_{n}(A)=f_{n}(C)=1$ for all $n<\omega$.

Although Theorem 2.3 shows that it is possible to remove the set-theoretic assumptions from Theorem 2.2 if $\mathcal{X}$ is a class which contains all bounded $p$-groups, this is not possible if $\mathcal{X}$ is a set as is required in [4, Problem 34]:

Corollary 2.6. (a) The following conditions are equivalent if both $A$ and $C$ are countable p-groups or the weak GCH holds:

(i) $f_{n}(A)=f_{n}(C)$ for all $n<\omega$.

(ii) $\operatorname{Hom}(A, G) \cong \operatorname{Hom}(C, G)$ for all cyclic p-groups $G$.

(iii) $\operatorname{char}(A) \cong \operatorname{char}(C)$.

(b) Let $\aleph_{\nu}$ be an uncountable cardinal number, and assume that Martin's Axiom and $2^{\aleph_{0}}>\aleph_{\nu}$ hold in addition to $Z F C$. There exist abelian p-groups $A$ and $C$ with $|A|,|C| \leq \aleph_{\nu}$ which satisfy $f_{0}(A) \neq f_{0}(C)$ and $\operatorname{Hom}(A, G) \cong \operatorname{Hom}(C, G)$ for all abelian groups $G$ with $|G| \leq \aleph_{\nu}$.

Proof. (a) It remains to consider the case that $A$ and $C$ are countable: The arguments of the proof of Theorem 2.3 can be used to establish $f_{n}(A)=f_{n}(C)$ if $f_{n}(A)$ or $f_{n}(C)$ is finite. Therefore, $f_{n}(A)$ and $f_{n}(C)$ are either both finite or both infinite. They have to coincide in the latter case since $f_{n}(A)$ and $f_{n}(C)$ are countable.

(b) Let $A=\bigoplus_{\aleph_{0}} \mathbb{Z}(p)$ and $C=\bigoplus_{\aleph_{1}} \mathbb{Z}(p)$. If $G[p]=0$, then $\operatorname{Hom}(A, G) \cong$ $\operatorname{Hom}(C, G)=0$. If $|G| \leq \aleph_{\nu}$ and $G[p] \neq 0$, then $\operatorname{Hom}(A, G) \cong \Pi_{\aleph_{0}} G[p]$ has cardinality $|G[p]|^{\aleph_{0}}=2^{\aleph_{0}}$ since $2^{\aleph_{0}}>\aleph_{\nu}$. On the other hand, $\operatorname{Hom}(C, G)$ has cardinality $|G[p]|^{\aleph_{1}}=2^{\aleph_{1}}$ for these $G$ by the same argument. By the MartinSolovay Theorem [5, Theorem 52], $2^{\aleph_{1}}=2^{\aleph_{0}}$, and $\operatorname{Hom}(A, G)$ and $\operatorname{Hom}(C, G)$ are isomorphic since both are $\mathbb{Z}(p)$-vector-spaces.

In particular, the abelian groups $A$ and $C$ given in the proof of part (b) of the last corollary have the following property: For a set $\mathcal{X}$ of abelian groups, choose an uncountable cardinal number $\aleph_{\nu}$ with $\aleph_{\nu}>\sup \{|G| \mid G \in \mathcal{X}\}$. By part (b) of the last corollary, we can find a model of ZFC in which $\operatorname{Hom}(A, G) \cong \operatorname{Hom}(C, G)$ for all $G \in \mathcal{X}$.

As a consequence of the last result, we obtain that the statement "Two $p$-groups $A$ and $C$ which are either torsion-complete or a direct sum of cyclics are isomorphic if and only if $\operatorname{char}(A) \cong \operatorname{char}(C)$ " is undecidable in ZFC.

\section{TORSION-FREE GROUPS}

In this section, we restrict our attention to the case in which we compare the groups $\operatorname{Hom}(A, B)$ and $\operatorname{Hom}(C, B)$ for $B$ chosen from a family of torsion-free abelian groups. The first result is categorical in nature:

Theorem 3.1. Let $R$ be an integral domain. Two R-modules $A$ and $C$ are isomorphic if and only if the modules $\operatorname{Hom}_{R}(A, B)$ and $\operatorname{Hom}_{R}(C, B)$ are naturally isomorphic for all $R$-modules $B$.

Proof. If $\phi: A \rightarrow C$ is an isomorphism, then the induced isomorphisms

$$
\operatorname{Hom}(\phi, B): \operatorname{Hom}_{R}(C, B) \rightarrow \operatorname{Hom}_{R}(A, B)
$$

are natural. 
Conversely, consider a collection $\left\{\Phi_{B}: \operatorname{Hom}_{R}(A, B) \rightarrow \operatorname{Hom}_{R}(C, B) \mid B\right.$ is an $R$-module $\}$ of natural isomorphisms. Let $\alpha=\Phi_{C}^{-1}\left(1_{C}\right)$ and $\beta=\Phi_{A}\left(1_{A}\right)$. By the naturality of the maps $\Phi$, the diagram

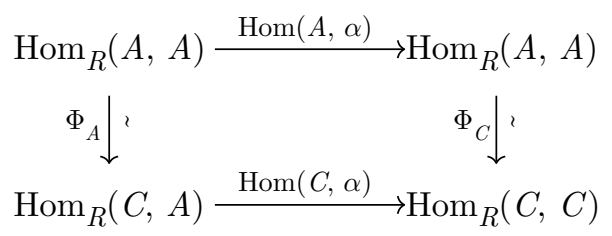

commutes. Thus, $\left[\Phi_{C} \operatorname{Hom}(A, \alpha)\right]\left(1_{A}\right)=\left[\operatorname{Hom}(C, \alpha) \Phi_{A}\left(1_{A}\right)\right.$, from which we obtain $]$

$$
1_{C}=\Phi_{C}(\alpha)=\Phi_{C} \operatorname{Hom}(A, \alpha)\left(1_{A}\right)=\operatorname{Hom}(C, \alpha) \Phi_{A}\left(1_{A}\right)=\alpha \beta .
$$

Since the maps $\Phi k_{B}^{-1}$ also form a natural collection of isomorphisms, we obtain $\beta \alpha=1$ in the same way.

Evidently, the last theorem carries over literally to the quasi-category of torsionfree modules over an integral domain $R$. However, we can improve it in this case by not only removing the naturality requirement on the isomorphism between $\operatorname{Hom}_{R}(A, B)$ and $\operatorname{Hom}_{R}(C, A)$, but also by restricting the ranks of the modules $B$ which need to be considered. We want to remind the reader that the rank of a torsion-free module $M$ over an integral domain $R$ is the dimension of the vectorspace $Q(R) \otimes_{R} M$ where $Q(R)$ is the field of quotients of $R$.

Theorem 3.2. Let $R$ be an integral domain, and $m<\omega$. Two torsion-free $R$ modules $A$ and $C$ of rank at most $m$ are quasi-isomorphic if and only if $\operatorname{Hom}_{R}(A, B)$ and $\operatorname{Hom}_{R}(C, B)$ are quasi-isomorphic for all torsion-free $R$-modules $B$ whose rank is at most $m$.

Proof. Let $A$ and $M$ be torsion-free $R$-modules whose ranks are at most $m$. It is enough to verify that $A$ and $C$ are quasi-isomorphic when $\operatorname{Hom}_{R}(A, B)$ and $\operatorname{Hom}_{R}(C, B)$ are quasi-isomorphic for each torsion-free module $B$ of rank not exceeding $m$. The $A$-socle of $M$, which is denoted by $S_{A}(M)$, is defined to be $\operatorname{Hom}_{R}(A, M) A$. Since $\operatorname{Hom}_{R}(A, C)=\operatorname{Hom}_{R}\left(A, S_{A}(C)\right)$, we have that $\operatorname{Hom}_{R}\left(C, S_{A}(C)\right)$ and $\operatorname{Hom}_{R}(C, C)$ are quasi-isomorphic $R$-modules. Observing that the module $\operatorname{Hom}_{R}(C, C)$ has finite rank, we obtain that $\operatorname{Hom}_{R}\left(C, S_{A}(C)\right)$ contains $d \operatorname{Hom}_{R}(C, C)$ for some non-zero $d \in R$. Therefore, $d 1_{C} \in \operatorname{Hom}_{R}\left(C, S_{A}(C)\right)$. This shows $d C \subseteq S_{A}(C)$. Likewise, $d^{\prime} A \subseteq S_{C}(A)$ for some non-zero $d^{\prime} \in R$. Without loss of generality, we may assume $A=A_{1} \oplus \cdots \oplus A_{n}$ and $C=C_{1} \oplus \cdots \oplus C_{m}$ where each $A_{j}$ and $C_{i}$ is a strongly indecomposable $R$-module since $Q(R) E_{R}(A)$ and $Q(R) E_{R}(C)$ are Artinian rings. Let $N$ be the nilradical of the endomorphism ring, $E_{R}(C)$, of $C$. We know that $N$ contains $\operatorname{Hom}_{R}\left(C_{i}, C_{j}\right)$ whenever $C_{i}$ is not quasi-isomorphic to $C_{j}$. Also, for quasi-isomorphic pairs $C_{i}$ and $C_{j}$, the ideal $N$ contains all those elements of $\operatorname{Hom}_{R}\left(C_{i}, C_{j}\right)$ which are not monomorphisms.

Since $N$ is nilpotent, $C / N C$ has positive torsion-free rank. If this were false, then $C / N^{k} C$ would be a torsion module for all $k<\omega$ which is not possible. Since $S_{C}(A) \doteq A$ and $S_{A}(C) \doteq C \nsubseteq N C$, there is a map $\phi: S_{C}(A) \rightarrow C$ such that $\operatorname{Im} \phi \nsubseteq \nsubseteq N C$. Since $S_{C}(A)=S_{C}\left(A_{1}\right) \oplus \cdots \oplus S_{C}\left(A_{n}\right)$, we may assume without loss of generality that $\phi\left(S_{C}\left(A_{1}\right)\right) \nsubseteq N C$. Moreover, $S_{C}\left(A_{1}\right)=\Sigma S_{C_{j}}\left(A_{1}\right)$ yields $\phi\left(S_{C_{j}}\left(A_{1}\right)\right) \nsubseteq N C$ for some $j$. Choose elements $c_{1}, \ldots, c_{r} \in C_{j}$ and $\gamma_{1}, \ldots, \gamma_{r} \in$ 
$\operatorname{Hom}_{R}\left(C_{j}, A_{1}\right)$ with $\phi\left(\sum_{i=1}^{r} \gamma_{i}\left(c_{i}\right)\right) \notin N C$. Evidently, not all the maps $\phi \gamma_{i}$ can be in $N$, say $\phi \gamma_{1} \notin N$.

Let $\pi_{i}: C \rightarrow C_{i}$ denote the natural projection. Observe that $\pi_{i} \phi \gamma_{1}$ is an endomorphism of $C$. However, not all the elements of the form $\pi_{i} \phi \gamma_{1}$ are in $N$, say $\pi_{1} \phi \gamma_{1} \notin N$. Since $\operatorname{Hom}_{R}\left(C_{1}, C_{j}\right) \subseteq N$ if $C_{1}$ and $C_{j}$ are not quasi-isomorphic, we obtain $C_{1} \sim C_{j}$ and the fact that $\pi_{1} \phi \gamma_{1}$ is a monomorphism. In particular, $\gamma_{1}$ has to be a monomorphism.

Since $C_{j} \sim C_{1}$, there is a monomorphism $\delta: C_{1} \rightarrow A_{1}$ such that $\pi_{1} \phi \delta \in$ $E\left(C_{1}\right)$ is a monomorphism. Therefore, $\pi_{1} \phi \delta$ is invertible in $Q(R) E_{R}\left(C_{1}\right)$ since $C_{1}$ is strongly indecomposable. This shows that $\delta$ quasi-splits. Since $A_{1}$ is strongly indecomposable, this is only possible if $A_{1} \sim C_{1}$.

For a torsion-free module $B$ of finite rank, we have

$$
\begin{aligned}
\operatorname{Hom}_{R}(A, B) & \sim \operatorname{Hom}_{R}\left(A_{1}, B\right) \oplus \operatorname{Hom}_{R}\left(A_{2} \oplus \cdots \oplus A_{n}, B\right) \\
& \sim \operatorname{Hom}_{R}\left(C_{1}, B\right) \oplus \operatorname{Hom}_{R}\left(C_{2} \oplus \cdots \oplus C_{n}, B\right)=\operatorname{Hom}_{R}(C, B) .
\end{aligned}
$$

By the Krull-Schmidt Theorem in the category of quasi-homomorphisms,

$$
\operatorname{Hom}_{R}\left(A_{2} \oplus \cdots \oplus A_{n}, B\right) \sim \operatorname{Hom}_{R}\left(C_{2} \oplus \cdots \oplus C_{m}, B\right) .
$$

An induction argument shows $A_{2} \oplus \cdots \oplus A_{n} \sim C_{2} \oplus \cdots \oplus C_{m}$.

Corollary 3.3. Let $R$ be an integral domain. Two torsion-free $R$-modules $A$ and $C$ of finite rank are quasi-isomorphic if and only if $\operatorname{Hom}_{R}(A, M) \sim \operatorname{Hom}_{R}(C, M)$ for all torsion-free R-modules (of finite rank).

If $A$ is a $J$-group, i.e. a torsion-free group of finite rank such that all groups $C \sim A$ are isomorphic to $A$, then we obtain

Corollary 3.4. Let $A$ be a J-group, and $C$ be a torsion-free group of finite rank. Then, $C \cong A$ if and only if $\operatorname{Hom}(A, B) \cong \operatorname{Hom}(C, B)$ for all torsion-free modules $B$ of finite rank.

However, considering the stronger requirement $\operatorname{Hom}(A, B) \cong \operatorname{Hom}(C, B)$ for all groups $B$ with $r_{0}(B) \leq m$ in Theorem 3.2 does not ensure that torsion-free abelian groups $A$ and $C$ or rank at most $m$ are isomorphic as the following example demonstrates. We want to remind the reader that every strongly indecomposable torsion-free group of rank 2 has a commutative endomorphism ring. It was shown in [3] that such a group is a $J$-group if and only it is a Murley group, i.e. $r_{p}(A) \leq 1$ for all primes $p$.

Example 3.5. There are torsion-free abelian groups $A$ and $C$ of rank 2 which are quasi-isomorphic, but not nearly isomorphic and satisfy $\operatorname{Hom}(A, B) \cong \operatorname{Hom}(C, B)$ for all torsion-free groups $B$ with $r_{0}(B) \leq 2$.

Proof. We may use the construction in [1] to find a homogeneous torsion-free group $A$ of rank 2 whose type is the type of $\mathbb{Z}_{p q}$, the localization of $\mathbb{Z}$ at the distinct primes $p$ and $q$, and has the following properties:

(a) $E(A)=\mathbb{Z}_{p q}$.

(b) Every rank 1 image of $A$ is isomorphic to $\mathbb{Z}_{p}$.

Having done this, we observe that $A$ is not a Murley group as mentioned above since $r_{p}(A)=2$. In particular, $A$ is not a $J$-group, and we can find a torsion-free group $C$ of rank 2 which is quasi-isomorphic, but not isomorphic to $A$. Since $A$ is semi-local, $C$ is not nearly isomorphic to $A$ either. Let $C$ be quasi-isomorphic 
to $A$, and consider a torsion-free abelian group $B$ of rank at most 2 . In the first instance, consider the case when rank $B=1$. If $B$ is not isomorphic to $\mathbb{Q}$ or $\mathbb{Z}_{p}$, then we have $\operatorname{Hom}(A, B)=\operatorname{Hom}(C, B)=0$. On the other hand, $\operatorname{Hom}\left(A, \mathbb{Z}_{p}\right)$ is a $\mathbb{Z}_{p}$-submodule of $\mathbb{Z}_{p} \oplus \mathbb{Z}_{p}$ since every rank 1 quotient of $A$ is isomorphic to $\mathbb{Z}_{p}$. This shows $\operatorname{Hom}\left(A, \mathbb{Z}_{p}\right) \cong \mathbb{Z}_{p} \oplus \mathbb{Z}_{p} \cong \operatorname{Hom}\left(C, \mathbb{Z}_{p}\right)$. Clearly, $\operatorname{Hom}(A, \mathbb{Z}) \cong \operatorname{Hom}(C, \mathbb{Q})$.

Now let $B$ have rank 2. Without loss of generality, we may assume that $B$ is indecomposable. If $\operatorname{Hom}(A, B) \neq 0$, then $S_{A}(B)$ has rank 1 or 2 . In the former case, we can apply the arguments of the previous paragraph to show $\operatorname{Hom}(A, B) \cong$ $\operatorname{Hom}(C, B)$ since $S_{C}(B)$ is quasi-equal to $S_{A}(B)$. Therefore, it remains to consider the case that $S_{A}(B)$ has rank 2. Then, $A$ embeds as a subgroup into $B$. Because $r_{p^{\prime}}(A) \leq 1$ for all primes $p^{\prime} \neq p$, we have that $C$ is isomorphic to a subgroup $C^{\prime}$ of $A$ whose index is a power of $p$. If $B$ is $p$-divisible, we have $\operatorname{Hom}(A, B) \cong \operatorname{Hom}(C, B)$. We, therefore, assume that $B$ is not $p$-divisible.

In the case that $q^{\omega} B \neq 0$, we observe that $B$ contains a pure subgroup $U$ isomorphic to $\mathbb{Z}_{p}$. Since every rank-1 quotient of $B$ contains one of $A$, the group $B / U$ is a $\mathbb{Z}_{p}$-module too. This implies that $B$ itself is a $\mathbb{Z}_{p}$-module. The sequence $0 \rightarrow \mathbb{Z}_{p q} \rightarrow A \stackrel{\alpha}{\rightarrow} \mathbb{Z}_{p} \rightarrow 0$ induces the exact sequence

$$
0 \rightarrow \operatorname{Hom}\left(\mathbb{Z}_{p}, B\right) \stackrel{\alpha_{*}}{\rightarrow} \operatorname{Hom}(A, B) \stackrel{\gamma}{\rightarrow} \operatorname{Hom}\left(\mathbb{Z}_{p q}, B\right) \rightarrow \operatorname{Ext}\left(\mathbb{Z}_{p}, B\right)
$$

for which $\operatorname{Ext}\left(\mathbb{Z}_{p}, B\right) \cong \operatorname{Ext}_{\mathbb{Z}_{p}}\left(\mathbb{Z}_{p}, B\right)=0$. Moreover, the groups $\operatorname{Hom}\left(\mathbb{Z}_{p}, B\right)$ and $\operatorname{Hom}\left(\mathbb{Z}_{p q}, B\right)$ are isomorphic to $B$.

Observe, the fact that $B$ is indecomposable guarantees that it is strongly indecomposable in this case. We want to remind the reader that a strongly indecomposable $p$-local group of rank 2 is a Murley group. In particular, $E(B)$ is a principal ideal domain. Choose an epimorphism $\beta: A \rightarrow \mathbb{Z}_{p}$ independent of $\alpha$. The map $\beta$ induces an embedding $\beta_{*}: \operatorname{Hom}\left(\mathbb{Z}_{p}, B\right) \rightarrow \operatorname{Hom}(A, B)$. If $g \in \operatorname{Im} \alpha_{*} \cap \operatorname{Im} \beta_{*}$, then $g=\gamma_{1} \alpha=\gamma_{2} \beta$ for some maps $\gamma_{i}: \mathbb{Z}_{p} \rightarrow B$. For non-zero elements $a \in$ ker $\alpha$ and $b \in \operatorname{ker} \beta$, we have $A=\langle a, b\rangle_{*}$. Therefore, $g(a)=0=g(b)$, and $g=0$. Define $\theta: \operatorname{Hom}\left(\mathbb{Z}_{p q}, B\right) \rightarrow \operatorname{Im} \beta_{*}$ to be any isomorphism. Since $\gamma \theta$ is a non-zero element of $E\left(\operatorname{Hom}\left(\mathbb{Z}_{p q}, B\right)\right) \cong E(B)$ and $E(B)$ is a principal ideal domain, this implies that $\gamma \theta$ is invertible in $Q E\left(\operatorname{Hom}\left(\mathbb{Z}_{p q}, B\right)\right)$. Therefore, the sequence $0 \rightarrow \operatorname{Hom}\left(\mathbb{Z}_{p}, B\right) \stackrel{\alpha_{*}}{\rightarrow}$ $\operatorname{Hom}(A, B) \stackrel{\gamma}{\rightarrow} \operatorname{Hom}\left(\mathbb{Z}_{p q}, B\right) \rightarrow 0$ quasi-splits. Since $\operatorname{Ext}(B, B)$ is torsion-free, the last sequence splits, i.e. $\operatorname{Hom}(A, B) \cong B \oplus B$. Now $\operatorname{Hom}(C, B) \sim B \oplus B$, so there is a quasi-splitting sequence $0 \rightarrow B_{1} \rightarrow \operatorname{Hom}(C, B) \rightarrow B_{2} \rightarrow 0$ with $B_{i} \sim B$. But $B$ is a $J$-group, and so $B_{i} \cong B$. As before, $\operatorname{Hom}(C, B) \cong B \oplus B$.

It remains to consider the case $q^{\omega} B=0$. We shall show that $\operatorname{Hom}(A, B)$ has rank 1. Since $\operatorname{Hom}(C, B)$ is quasi-isomorphic to $\operatorname{Hom}(A, B)$, these two groups have to be isomorphic as well. Set $F$ to be a subgroup of $A$ for which $A / F \cong \mathbb{Z}\left(q^{\infty}\right)$. We then observe that $F$ is a free $\mathbb{Z}_{p q}$-module, and that $A / F \subseteq B / F \subseteq \mathbb{Q} A / F \cong$ $\mathbb{Z}^{2}\left(p^{\infty}\right) \oplus \mathbb{Z}^{2}\left(q^{\infty}\right)$. In the case under consideration, $B / A$ does not contain a subgroup isomorphic to $\mathbb{Z}\left(q^{\infty}\right)$. If $B / A$ is finite, then $A \sim B$ and $\operatorname{Hom}(A, B) \cong \mathbb{Z}_{p q}$. If $B / A$ contains $\mathbb{Z}^{2}\left(p^{\infty}\right)$, then $p^{\omega} B=B$, a case which has been settled earlier. Thus, we may assume that $B / A \cong \mathbb{Z}\left(p^{\infty}\right) \oplus T$ where $T$ is finite. Let $\phi \in \operatorname{Hom}(A, B)$. Since neither $A$ nor $\phi(A)$ map onto $\mathbb{Z}\left(p^{\infty}\right)$, the subgroup $(\phi(A)+A) / A$ of $B / A$ must be finite. For some non-zero integer $n$, we have $n \phi(A) \subseteq A$. In particular, $n \phi \in E(A)$, and $\operatorname{Hom}(A, B)$ has rank 1. 
We conclude this paper with a result which shows that the consideration of groups of the form $\operatorname{Hom}(A, T)$ with $T$ torsion does not yield any further information beyond what has been already obtained.

Proposition 3.6. Let $p$ be a prime. The following conditions are equivalent for torsion-free abelian groups $A$ and $C$ of finite rank:

(a) $r_{p}(A)=r_{p}(C)$; and $r_{0}(A)=r_{p}(A)$ if and only if $r_{0}(C)=r_{p}(C)$.

(b) $\operatorname{Hom}(A, G) \cong \operatorname{Hom}(C, G)$ for all p-groups $G$.

Proof. (a) $\Rightarrow$ (b): Let $G$ be a $p$-group, and $F$ a $p$-basic subgroup of $A$. There is an induced exact sequence $0 \rightarrow F_{p} \rightarrow A_{p} \rightarrow H \rightarrow 0$ in which $H$ is a torsionfree divisible group whose rank is $m=r_{0}(A / F)=r_{0}(A)-r_{p}(A)$. This sequence induces the exact sequence $0 \rightarrow \operatorname{Hom}\left(\bigoplus_{m} \mathbb{Q}, G\right) \rightarrow \operatorname{Hom}\left(A_{p}, G\right) \rightarrow \operatorname{Hom}\left(F_{p}, G\right) \rightarrow$ $\operatorname{Ext}\left(\bigoplus_{m} \mathbb{Q}, G\right)$. Since $\operatorname{Hom}\left(\bigoplus_{m} \mathbb{Q}, G\right)$ and $\operatorname{Ext}\left(\bigoplus_{m} \mathbb{Q}, G\right)$ are torsion-free divisible, the short exact sequence $0 \rightarrow \operatorname{Hom}\left(\bigoplus_{m} \mathbb{Q}, G\right) \rightarrow \operatorname{Hom}\left(A_{p}, G\right) \rightarrow \operatorname{Hom}\left(F_{p}, G\right) \rightarrow 0$ splits. Thus, $\operatorname{Hom}\left(A_{p}, G\right) \cong \operatorname{Hom}\left(\bigoplus_{m} \mathbb{Q}, G\right) \oplus \bigoplus_{r_{p}(A)} G$.

Suppose that $m \neq 0$. Then, $r_{0}\left(\operatorname{Hom}\left(\bigoplus_{m} \mathbb{Q}, G\right)\right)=m r_{0}(\operatorname{Hom}(\mathbb{Q}, G))$. If $G$ is reduced, then $\operatorname{Hom}(\mathbb{Q}, G)=0$. Otherwise, $\operatorname{Hom}(\mathbb{Q}, G)$ contains a direct summand isomorphic to $\operatorname{Hom}\left(\mathbb{Q}, \mathbb{Z}\left(p^{\infty}\right)\right)$ which has torsion-free rank $2^{\aleph_{0}}$. Consequently,

$$
\operatorname{Hom}\left(A_{p}, G\right) \cong \begin{cases}\bigoplus_{r_{p}(A)} G & \text { if } r_{0}(A)=r_{p}(A), \\ \operatorname{Hom}(\mathbb{Q}, G) \oplus \bigoplus_{r_{p}(A)} G & \text { otherwise. }\end{cases}
$$

This shows $\operatorname{Hom}\left(A_{p}, G\right) \cong \operatorname{Hom}\left(C_{p}, G\right)$. On the other hand, there is an exact sequence $0 \rightarrow A \rightarrow A_{p} \rightarrow H^{\prime} \rightarrow 0$ in which $H^{\prime}$ is a divisible torsion group with $H^{\prime}[p]=0$. This induces the exact sequence $0=\operatorname{Hom}\left(H^{\prime}, G\right) \rightarrow \operatorname{Hom}\left(A_{p}, G\right) \rightarrow$ $\operatorname{Hom}(A, G) \rightarrow \operatorname{Ext}\left(H^{\prime}, G\right)=0$.

(b) $\Rightarrow$ (a): Observe $\operatorname{Hom}(A, \mathbb{Z}(p)) \cong A / p A \cong \bigoplus_{r_{p}(A)} \mathbb{Z}(p)$. Therefore, $r_{p}(A)=$ $r_{p}(C)$. Assume $r_{p}(A)<r_{0}(A)$, but $r_{p}(C)=r_{0}(C)$. Using the arguments from the proof of the previous implication, we obtain that $\operatorname{Hom}\left(\mathbb{Q}, \mathbb{Z}\left(p^{\infty}\right)\right)$ is a direct summand of $\operatorname{Hom}\left(A, \mathbb{Z}\left(p^{\infty}\right)\right)$ while $\operatorname{Hom}\left(C, \mathbb{Z}\left(p^{\infty}\right)\right)$ is a torsion group. This proves the proposition.

\section{REFERENCES}

1. R. A. Beaumont and R. S. Pierce, Torsion-free groups of rank two, Memoirs of the AMS, Vol. 38 (1961). MR 24:A162

2. R. A. Beaumont and R. J. Wisner, Rings with additive group which is torsion-free of rank two, Acta. Sci. Math. (Szeged) 20 (1959), 105-116. MR 21:5651

3. T. Faticoni and H. P. Goeters, On torsion-free Ext, Comm. in Algebra 16(9) (1988), 18531876. MR 90d:20098

4. L. Fuchs, Infinite Abelian Groups, Vol. I/II, Academic Press, New York, London (1970/73). MR 41:333; MR 50:2362

5. T. Jech, Set Theory, Academic Press, New York, London (1978). MR 80a:03062

Department of Mathematics, Auburn University, Auburn, Alabama 36849

E-mail address: albreuf@mail.auburn.edu

E-mail address: goetehp@mail.auburn.edu 